\title{
Source compacte de rayons $X$ basée sur la résonance cyclotronique électronique
}

\author{
C. Gaudin, C. Rouillé, K. Serebrennikov, J.M. Buzzi, M. Bacal, \\ M. Lamoureux* et P. Charles*
}

\section{Laboratoire de Physique des Milieux Ionisés, UMR 7648, École Polytechnique, 91128 Palaiseau cedex, France \\ * Laboratoire de Dynamique des lons, Atomes et Molécules, Case 75, Université Pierre et Marie Curie, 4 place Jussieu, 75252 Paris, France}

\begin{abstract}
Résumé : Une source ECR compacte a été construite en vue de produire des rayons X pour des applications médicales et technologiques. Le plasma est créé par une micro-onde $(2,45 \mathrm{GHz})$ injectée dans un miroir magnétique et un anneau d'électrons chauds a été repéré dans le plan médian. L'analyse des spectres de rayonnement de freinage émis par le plasma a montré que le paramètre de température de ces électrons pouvait atteindre $20 \mathrm{keV}$. Une cible a été introduite au voisinage de l'anneau pour obtenir un débit de dose satisfaisant.
\end{abstract}

\section{INTRODUCTION ET PRESENTATION DU DISPOSITIF EXPERIMENTAL}

Les sources de plasma fonctionnant à la résonance cyclotronique électronique (ECR) sont couramment utilisées pour la production des ions multichargés et sont connues aussi pour produire des électrons chauds. Dans notre source compacte, nous utilisons ces électrons pour émettre des rayons X avec une énergie maximale d'une cinquantaine de $\mathrm{keV}$, ce qui est compatible avec les besoins de la radiologie médicale [1] ou industrielle.

Notre source est présentée sur la figure 1. Le confinement des particules est obtenu par un miroir magnétique réalisé par des aimants permanents dans une enceinte cylindrique en aluminium de 1 litre. Le champ magnétique maximal que nous pouvons obtenir au centre de l'enceinte est de $0,19 \mathrm{~T}$. La distance entre les aimants (L) peut être changée afin de choisir la configuration magnétique. Le plasma et les électrons énergétiques sont créés grâce à l'injection d'une micro-onde de fréquence $2,45 \mathrm{GHz}$ par un guide rectangulaire. La pression de travail varie entre $5.10^{-5}$ et $10^{-4}$ Torr. Tous ces éléments rendent la source compacte. Les rayons $X$ produits sont filtrés par une fenêtre en aluminium de $0,2 \mathrm{~mm}$ d'épaisseur qui élimine les photons de basses énergies du faisceau $(<10 \mathrm{keV})$.

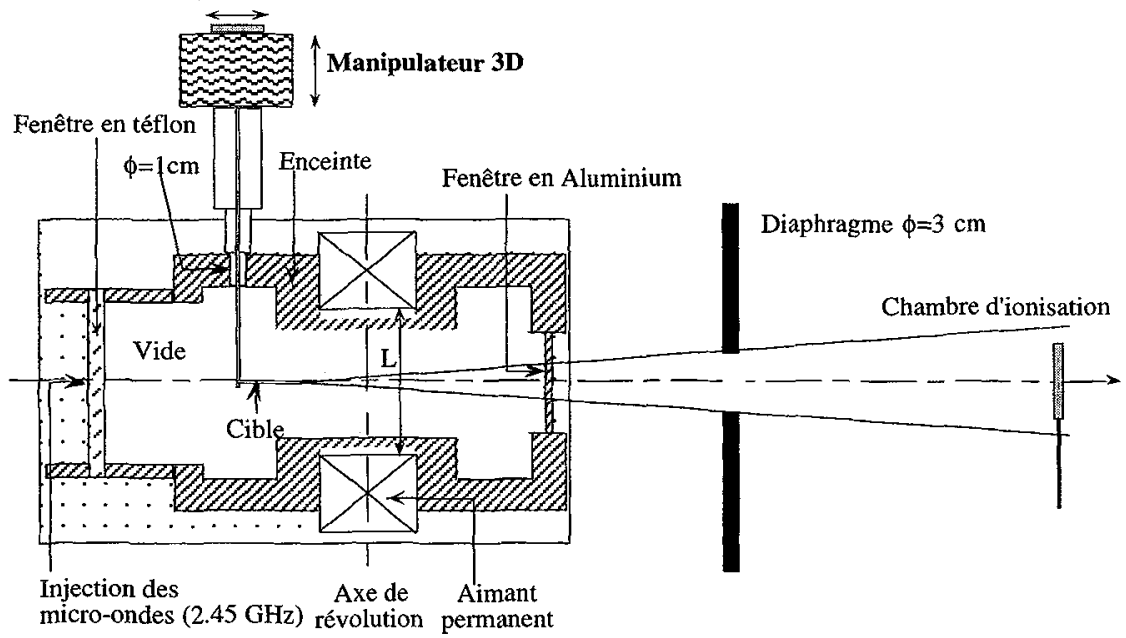

Figure 1 : Présentation du dispositif expérimental

\section{ETUDE DES ELECTRONS CHAUDS ET DU FAISCEAU DE RAYONS X}

Les premières études ont montré l'existence dans ce type de source, utilisant des champs magnétiques faibles $(<1 \mathrm{kG})$, d'un anneau d'électrons énergétiques $(>10 \mathrm{keV})$ dans le plan médian du miroir [2]. Afin 
de déterminer leur température, un diagnostic [3] développé pour les sources ECRIS et basé sur l'analyse des spectres de rayonnement de freinage obtenus sur le gaz, a été adapté aux conditions d'observation et à l'énergie des photons de notre source. Nous montrons que le paramètre de température des électrons de l'anneau augmente faiblement avec la puissance micro-onde [2] et que la configuration magnétique joue un rôle fondamental pour l'obtention d'une forte densité d'électrons énergétiques. La température passe de $5 \mathrm{keV}$ à $20 \mathrm{keV}$ lorsque l'homogénéité du champ magnétique augmente, et quelques électrons atteignent alors $100 \mathrm{keV}$. L'intensité globale du rayonnement est décuplée. Nous avons donc déterminé les conditions permettant d'optimiser le paramètre de température et la densité des électrons chauds pour ce plasma.

Pour atteindre les intensités nécessaires en radiologie, l'utilisation d'une cible est obligatoire. Une cible en tungstène, montée sur un manipulateur (cf. Fig. 1) a été placée au voisinage de l'anneau d'électrons. Le faisceau a été caractérisé en intensité grâce à une chambre d'ionisation Keithley. Cette chambre mesure le débit de dose $(\mathrm{Gy} / \mathrm{s})$ et se trouve à $80 \mathrm{~cm}$ du foyer pour se placer dans des conditions similaires à celles de la radiologie. Le Gray $(\mathrm{J} / \mathrm{kg})$ est une unité couramment employée dans le domaine médical ce qui nous permet de faire des comparaisons entre les tubes de rayons $X$ et notre source. Nous n'avons pas étudié la source pour les faibles puissances $(<400 \mathrm{~W})$. La position de la cible est très importante, elle ne doit pas perturber l'anneau et doit conduire à une intensité des rayons $\mathrm{X}$ suffisante. En effet, sur la figure 2 nous pouvons voir que, lorsque la cible est juste en limite de l'anneau (position 1 ou optimale sur la figure), le débit de dose est 10 fois plus grand que lorsqu'elle l'intercepte largement le faisceau (position 2). La figure 2 montre également qu'il est très facile de régler l'intensité du faisceau en changeant la puissance micro-onde, la variation de l'énergie restant faible.

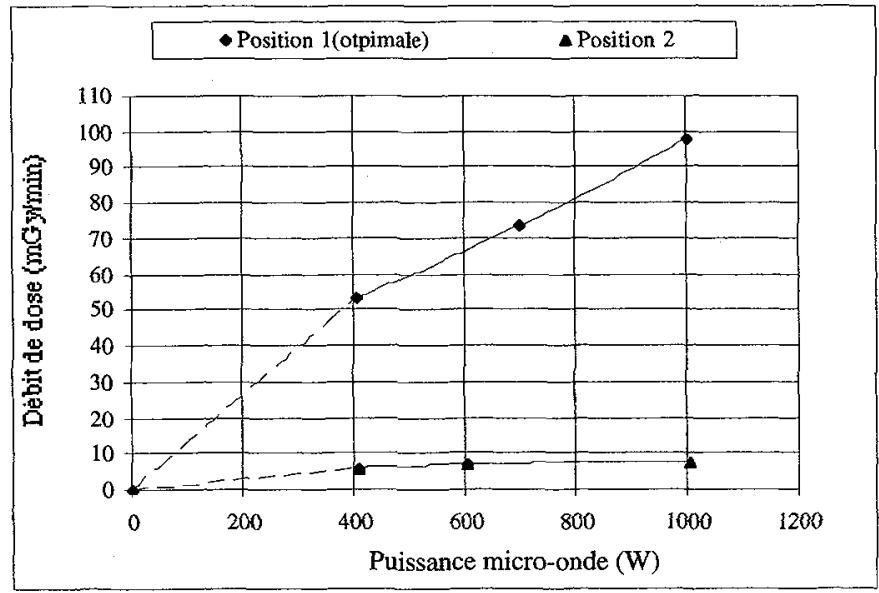

Figure 2: Variation du débit de dose du faisceau de rayons $\mathrm{X}$ en fonction de la puissance micro-onde incidente pour deux positions de la cible par rapport à l'anneau d'électrons chauds.

Le débit de dose maximal que nous pouvons obtenir est de $9 \mathrm{cGy} / \mathrm{mn}$. Ces résultats sont compatibles avec les débits de dose requis pour la radioscopie et certains types de radiographies.

\section{Remerciements}

Un des auteurs (C. G.) tient à remercier la DRET pour le soutien financier, contrat DRET/96/1200/A00.

\section{Références}

1. M. Bacal, C. Gaudin, A. Bourdier, J. Bruneteau, J-M. Buzzi, K.S. Golovanivsky, L. Hay, C. Rouillé, Nature (London) 384, 421 (1996).

2. C. Gaudin, L. Hay, J-M. Buzzi, M. Bacal, M. Lamoureux., Rev. Sci. Instr. 69, 890 (1998).

3. C. Barué, M. Lamoureux, P. Briand, A. Girard, et G. Melin, J. Appl. Phys. 76, 2662 (1994). R. Pras, Thèse Paris VI (1997). 